\title{
RELATIVIZED WEAK DISJOINTNESS AND RELATIVELY INVARIANT MEASURES
}

BY

\author{
DOUGLAS C. MCMAHON
}

\begin{abstract}
In this paper we study the relativized weak disjointness and the relativized regionally proximal relation for homomorphisms of point-transitive transformation groups, under the assumption of a relativized invariant measure. We also include a proof of a Folner-type result for syndetic subsets of an amenable group.
\end{abstract}

Introduction. In [P] R. Peleg proves the following:

If $(X, T)$ and $(Y, T)$ are metric minimal transformation groups supporting invariant measures then $(X, T)$ and $(Y, T)$ are weakly disjoint $((X \times Y, T)$ has a point with dense orbit) iff their maximal equicontinuous factors $(X / S, T)$ and $(Y / S, T)$ are disjoint.

Motivated by this result Glasner conjectures at the end of [G] that

If $(X, T) \rightarrow^{\Phi}(Y, T)$ supports a relatively invariant measure where $X$ is minimal and metric, then $R(\varphi)=\left\{\left(x, x^{\prime}\right) \in X \times X \mid \varphi(x)=\varphi\left(x^{\prime}\right)\right\}$ has a point with dense orbit iff the only almost periodic extension of $Y$ which is a factor of $X$ is $Y$ itself.

The only if part of the above is easily seen to be true. Example 3.2.1 of this paper shows that the if part is not true without some further restriction on $\varphi$.

One possible restriction on $\varphi$ is to assume that it is open. With this additional condition we prove the following variation of Glasner's conjecture (see 1.9). Suppose $X$ and $Y$ are minimal and metric, the natural projection $X \rightarrow X / S(\varphi)$ is open (where $S(\varphi)$ is the relativized equicontinuous structure relation), $\theta$ has a relative invariant measure, and $X / S(\varphi)$ and $Y / S(\theta)$ are relatively disjoint $(X / S(\varphi) \circ z Y / S(\theta)=\{(x / S(\varphi), y / S(\theta)): \varphi(x)=$ $\theta(y)\}$ is a minimal set). Fix $x$ in $X$. Then for some nonempty compact set, $B(x)$, contained in $Y$, the set $D(x)=\{y \in B(x):(x, y)$ has a dense orbit in $\left.X \circ{ }^{Z} Y\right\}$ is a dense $G_{\delta}$ subset of $B(X)$.

When $Z$ is a singleton, the restriction that $X \rightarrow X / S(\varphi)$ is open can be dropped, thus giving us a generalization of Peleg's theorem.

We also show that if $X$ is minimal and $\varphi: X \rightarrow Z$ has a relatively invariant measure and if $z \in Z$, then there exists a compact set $B_{z} \subseteq X$ such that for $x$

Received by the editors October 29, 1976.

AMS (MOS) subject classifications (1970). Primary 54H20.

Key words and phrases. Relativized, weak disjointness, RIM, point-transitive. 
in $B_{z}, S(\varphi)(x)=Q(\varphi)(x)$ and moreover for $x^{\prime} \in S(\varphi)(x)$, there exist nets $t_{n}$ in $T$ and $x_{n}$ in $B_{z}$ such that $x_{n} \rightarrow x, x_{n} t_{n} \rightarrow x$, and $x^{\prime} t_{n} \rightarrow x$ (see 1.5).

These results are contained in $\$ 1$ where we study relative invariant measures for homomorphisms between point-transitive transformation groups. The main technique used in $\$ 1$ was motivated by Lemma 3 of Pelleg's paper $[\mathrm{P}]$ that shows that if $(X, \mu),(Y, v)$ are probability spaces and $(Y, v)$ is separable then the following conditions on a measurable subset $E \subseteq X \times Y$ are equivalent.

(1) $E$ is a rectangle (a.e. $\mu \times v$ ) of the form $X \times B$.

(2) $v\left(E_{x}\right)=C$ a.e. $\mu$ and $v\left(E_{x} \cap E_{s}\right)=C^{\prime}$ a.e. $\mu \times \mu\left(E_{x}\right.$ denotes the section at $x$ ).

$\$ 2$ contains some results about the relativized equicontinuous structure relation, $S(\varphi)$, under the assumption that the almost periodic points in $R(\varphi)$ are dense in $R(\varphi)$. Some of these results are known (see [MW]), however, the proofs are new and provide a nice application of $\$ 1$.

$\$ 3$ contains examples. $\$ 4$ contains a result for syndetic subsets of amenable groups similar to results in [EK] and [F], see [EK] and [F] for a more thorough discussion of the results.

I would like to thank T. S. Wu for reading portions of the rough draft and making helpful comments on it.

The referee pointed out that in a paper he was refereeing at the same time, Furstenberg and Glasner gave an entirely different proof of the Glasner conjecture (with the "open" hypothesis). Their proof is from the viewpoint of ergodic theory, [FG].

In response to a question to the referee I have included a proof of the Glasner conjecture (with the "open" hypothesis) in the nonmetric case, the conclusion being that $R(\varphi)$ is topologically transitive-that is, every nonempty open invariant set is dense (this is equivalent to having a point with dense orbit in the metric case), see 1.8 and 1.9.

Preliminaries. Let $(X, T)$ be a transformation group with compact Hausdorff phase space. We will write $X$ for both the transformation group $(X, T)$ and the phase space. If $X$ is point-transitive, let $X_{m}$ denote the set of transitive points in $X$; when $X$ is metric, $X_{m}$ is a dense $G_{8}$ set. For a homomorphism $\varphi$ of $X$ onto $Y, R_{m}(\varphi)=\left\{\left(x, x^{\prime}\right) \in X_{m} \times X_{m}: \varphi(x)=\varphi\left(x^{\prime}\right)\right\}, P_{m}(\varphi)=\left\{\left(x, x^{\prime}\right)\right.$ $\in X_{m}$ : there exists a net $t_{n}$ in $T$ with $\left.x t_{n} \rightarrow x_{0}, x^{\prime} t_{n} \rightarrow x_{0}\right\}$, for any $x_{0}$ in $X_{n}$; $Q_{m}(\varphi)=\left\{\left(x, x^{\prime}\right) \in R_{m}(\varphi)\right.$ : there exist nets $t_{n}$ in $T$ and $\left(x_{n}, x_{n}^{\prime}\right)$ in $R_{m}(\varphi)$ such that $\left(x_{n}, x_{n}^{\prime}\right) \rightarrow\left(x, x^{\prime}\right)$ and $\left.\left(x_{n}, x_{n}^{\prime}\right) t_{n} \rightarrow\left(x_{0}, x_{0}^{\prime}\right)\right\}$ for any $x_{0}$ in $X_{m} ; S_{m}(\varphi)$ is the smallest closed (in $R_{m}(\varphi)$ ) invariant equivalence relation containing $Q_{m}(\varphi)$.

Let $D_{m}(\varphi)$ be the set of almost periodic points in $R_{m}(\varphi)$. When $X$ is minimal, the subscript $m$ is omitted. When $Y$ is a singleton, $\varphi$ is omitted.

Consider $T$ with the discrete topology, let $M$ be a minimal set in $\beta T$ and let 
$J \cdot$ be the set of idempotents in $M$, see [E] or [ $\left.\mathbf{G}_{b}\right]$ for properties of $\beta T$ and $M$.

Let $M(X)$ be the set of Borel probability measures on $X$. For $\mu$ in $M(X)$ define $\mu t$ by $\mu t(A)=\mu\left(A t^{-1}\right)$ for every measurable set $A$.

Defintmon. A section $\lambda$ for $\varphi: X \rightarrow Y$ is a homomorphism $\lambda: Y \rightarrow M(X)$ such that $\hat{\varphi}\left(\lambda_{y}\right)=\delta_{y}$ where $\hat{\varphi}\left(\lambda_{y}\right)(A)=\lambda_{y} \varphi^{-1}(A)$ for every Borel subset $A$ of $Y$ and $\delta$ is the point mass at $y$.

Notation. $(\varphi, \lambda) \in \mathcal{S}$ will denote that $\lambda$ is a section for $\varphi$, and $B(x)$ will denote the support of $\lambda_{\varphi(x)}$, for $x$ in $X$.

REMARK. (1) Since $\lambda$ is a homomorphism $\lambda_{y} t=\lambda_{y, t}$, so $\lambda_{y t}(A t)=\left(\lambda_{y} t\right)(A t)=$

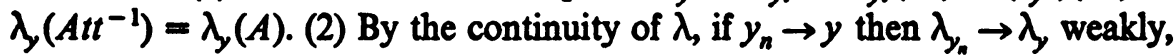
which means $\int f d \lambda_{,} \rightarrow \int f d \lambda$, for every continuous real-valued function. This is equivalent to $\lambda_{y_{A}}(A) \rightarrow \lambda_{y}(A)$ for every Borel set $A$ with $\lambda_{y}(\partial A)=0$, where $\partial A=\operatorname{cls}(A) \backslash \operatorname{int}(A)$ is the boundary of $A$. Note also if $F$ is a closed set, $\lambda_{y}(F)=\inf \left\{\lambda_{y}(V): V\right.$ is an open set containing $F$ with $\left.\lambda_{y}(\partial V)=0\right\}$. A set $V$ with $\lambda_{y}(\partial V)=0$ is called a continuity set (for $\left.\lambda_{y}\right)$. We will use the notation $\tau(F)=\tau_{\lambda}(F)=\Re_{y}(F)$ to denote the set of open continuity sets (for $\lambda_{y}$ ) containing $F$. Note that if $(\theta, \lambda) \in \delta, \varepsilon>0, z \in Z$, and $V$ is a continuity set of $\lambda$, then there is a neighborhood $U$ of $z$ such that $\left|\lambda_{z}(V)-\lambda_{z}(V)\right|<\varepsilon$ for $z^{\prime}$ in $U$.

We will be considering the following situation. $X, Y, Z$ are point-transitive transformation groups and are compact Hausdorff; $X_{m}, Y_{m}, Z_{m}$ are the sets of transitive points in $X, Y, Z$ respectively, they are nonempty by assumption, $\varphi$ : $X \rightarrow Z$ and $\theta: Y \rightarrow Z$ are homomorphisms onto $Z$. Let $X{ }^{Z} Z Y=\{(x, y)$ : $\varphi(x)=\theta(y)\}$; we write $X \perp^{z} Y$ and say $X$ is disjoint from $Y$ over $Z$, if $X{ }^{Z} Y$ is minimal; we write $X \dot{ }^{Z} Y$ and say $X$ is weakly disjoint from $Y$ over $Z$, if $X \bullet^{Z} Y$ is point-transitive. If $N$ is a subset of $X \bullet^{Z} Y$ let $N_{x}=\{y$ : $(x, y) \in N\}$.

1. It may be helpful to first read the results in this section assuming $X$ and $Y$ are minimal, in which case the subscript " $m$ " may be dropped.

(1.1) Lemar. Given $(\theta, \lambda) \in \delta$. If $N$ is a closed invariant subset of $X{ }^{Z} Y$, then $\lambda_{\varphi(x)}\left(N_{x}\right)=\lambda_{\varphi(x)}\left(N_{x^{\prime}}\right)$ for every $x, x^{\prime}$ in $X_{m}$.

Proor. For any $\varepsilon>0$, take $V \in \Re\left(N_{x}\right)$ such that $\lambda_{\varphi(x)}(V)<\lambda_{\varphi(x)}\left(N_{x}\right)+$ e. Take a neighborhood $U$ of $z=\phi(x)$ such that $\left|\lambda_{z}(V)-\lambda_{z}(V)\right|<\varepsilon$ for $z^{\prime}$ in $U$. Let $t_{n}$ be a net in $T$ with $x^{\prime} t_{n} \rightarrow x$. Then $N_{x^{\prime}} t_{n} \subseteq V$ and $\varphi(x) t_{n} \in U$ for some $t_{n}$, since otherwise there would exist $y_{n}$ in $N_{x^{\prime}}$ such that $y_{n} t_{n} \notin V$ and some subnet $y_{m} t_{m}$ converges to $y \notin V$ since $Y$ is compact, but $\left(x^{\prime}, y_{m}\right) t_{m} \in N$ and so $(x, y) \in N$ and $y \in N_{x} \subseteq V$, a contradiction. For such a $t_{n}, \lambda_{\varphi(x)}\left(N_{x}\right)$ $=\lambda_{\varphi(x) t_{n}}\left(N_{x^{\prime}} t_{n}\right)<\lambda_{\varphi(x) t_{n}}(V)<\lambda_{\varphi(x)}(v)+\varepsilon<\lambda_{\varphi(x)}\left(N_{x}\right)+2 \varepsilon$. Thus $\lambda_{\varphi(x)}\left(N_{x^{\prime}}\right)$ $<\lambda_{\varphi(x)}\left(N_{x}\right)$, since $\varepsilon$ was arbitrary. The lemma follows by symmetry. 
(1.2) Lemma. Given $(\theta, \lambda) \in \mathcal{S}$ and $N$ a closed invariant subset of $X \bullet^{Z} Y$. Let $K=K(N)=\left\{\left(x, x^{\prime}\right) \in R_{m}(\varphi): \lambda_{\varphi(x)}\left(N_{x} \Delta N_{x^{\prime}}\right)=0\right\}$. Then $K$ is a closed (in $X_{m} \times X_{m}$ ) invariant equivalence relation on $X_{m}$ containing $Q_{m}(\varphi)$. In addition if $\left(x_{0}, x_{n}\right) \in K, n=1,2,3, \ldots$, then

$$
\begin{aligned}
\lambda_{\varphi\left(x_{0}\right)}\left(\cap\left\{N_{x_{n}}: n=0,1,2, \ldots\right\}\right) & =\lambda_{\varphi\left(x_{0}\right)}\left(N_{x_{0}}\right) \\
& =\lambda_{\varphi\left(x_{0}\right)}\left(\cup\left\{N_{x_{n}}: n=0,1, \ldots\right\}\right) .
\end{aligned}
$$

Note $\left(x, x^{\prime}\right) \in K$ iff $\lambda_{\varphi(x)}\left(N_{x} \cap N_{x^{\prime}}\right)=\lambda_{\varphi(x)}\left(N_{x}\right)$ iff $\lambda_{\varphi(x)}\left(N_{x} \cup N_{x^{\prime}}\right)=$ $\lambda_{\varphi(x)}\left(N_{x}\right)$.

Proof. We first prove the following two sublemmas.

Lemana. Suppose $N$ is a closed invariant subset of $X \circ^{z} Y,(\theta, \lambda) \in \delta$, $p \in X_{m}$, and $U \in \mathcal{R}\left(N_{p}\right)$ with $\lambda_{\varphi(p)}\left(U \backslash N_{p}\right)<\varepsilon$. Then for $q$ in $X_{m}$ sufficiently close to $p, N_{q} \subseteq U$ and $\lambda_{\varphi(q)}\left(U \backslash N_{q}\right)<\varepsilon$.

Proof. Clearly $N_{q} \subseteq U$ for $q$ sufficiently close to $p$. Now take $\delta$ so that $0<\delta<\varepsilon-\lambda_{\varphi(p)}\left(U \backslash N_{p}\right)$ and note that for $q$ sufficiently close to $p, \mid \lambda_{\varphi(q)}(U)$ $-\lambda_{\phi(p)}(U) \mid<\delta$. So

$$
\begin{aligned}
\lambda_{\varphi(q)}\left(U \backslash N_{q}\right) & =\lambda_{\varphi(q)}(U)-\lambda_{\varphi(q)}\left(N_{q}\right) \\
& <\lambda_{\varphi(p)}(U)+\delta-\lambda_{\varphi(p)}\left(N_{p}\right)=\lambda_{\varphi(p)}\left(U \backslash N_{p}\right)+\delta<\varepsilon .
\end{aligned}
$$

Leman. Suppose $\lambda$ is a probability measure, $A \subseteq B, C \subseteq D, \lambda(B \backslash A)<\varepsilon$ and $\lambda(D \backslash C)<\varepsilon$. Then $|\lambda(C \backslash A)-\lambda(D \backslash B)|<\varepsilon$.

Proof. Note $B=A \cup(B \backslash A)$ so $B \backslash D=(A \backslash D) \cup[B \backslash(A \cup D)]$ and $0<\lambda(B \backslash D)-\lambda(A \backslash D)=\lambda(B \backslash(A \cup D))<\varepsilon$. Also note $A \backslash C=(A \backslash$ $D) \cup(D \backslash C)$, so $0<\lambda(A \backslash C)-\lambda(A \backslash D)=\lambda(D \backslash C)<\varepsilon$. Thus $\mid \lambda(B \backslash D)$ $-\lambda(A \backslash C) \mid<\varepsilon$.

Proof OF Lemana 1.2. Clearly $K$ is an equivalence relation. Note that $\lambda_{\varphi(p)}\left(N_{p} \Delta N_{q}\right)=2 \lambda_{\varphi(p)}\left(N_{p} \backslash N_{q}\right)$ for $(p, q) \in R_{m}(\varphi)$.

Now we will show that $K$ contains $Q_{m}(\varphi)$. Suppose $(p, q) \in Q_{m}(\varphi)$, note $\lambda_{\varphi(p)}=\lambda_{\varphi(q)}$. Given any $\varepsilon>0$, take $U \in \mathcal{R}\left(N_{p}\right), V \in \mathcal{R}\left(N_{q}\right)$ such that $\lambda_{\varphi(p)}\left(U \backslash N_{p}\right)<\varepsilon$ and $\lambda_{\varphi(p)}\left(V \backslash N_{q}\right)<\varepsilon$, and so (i) $\mid \lambda_{\varphi(p)}(U \backslash V)-\lambda_{\varphi(p)}\left(N_{p} \backslash\right.$ $\left.N_{q}\right) \mid<\varepsilon$. Now we can take $\left(p^{\prime}, q^{\prime}\right) \in R_{m}(\varphi)$ and $t$ in $T$ such that $N_{p^{\prime}} \subseteq U$, $N_{q^{\prime}} \subseteq V, \lambda_{\varphi(p)}\left(U \backslash N_{p^{\prime}}\right)<\varepsilon, \lambda_{\varphi(p)}\left(V \backslash N_{q^{\prime}}\right)<\varepsilon$, (and so (ii) $\lambda_{\varphi(p)}(U \backslash V)-$ $\left.\lambda_{\varphi(p)}\left(N_{p^{\prime}} \backslash N_{q^{\prime}}\right) \mid<\varepsilon\right)$; (iii) $\left|\lambda_{\varphi(p)}(U \backslash V)-\lambda_{\varphi(p)}(U \backslash V)\right|<\varepsilon$; and $N_{p^{\prime} t} \subseteq U$, $N_{q^{\prime} t} \subseteq U, \lambda_{\varphi\left(p^{\prime}\right)}\left(U \backslash N_{p^{\prime} t}\right)<\varepsilon, \lambda_{\varphi\left(p^{\prime} t\right)}\left(U \backslash N_{q^{\prime} t}\right)<\varepsilon$, (and so (iv) $\lambda_{\varphi(p)}\left(N_{p^{\prime}} \backslash\right.$ $\left.\left.N_{q^{\prime}}\right)=\lambda_{\left(p^{\prime} t\right)}\left(N_{p^{\prime} t} \backslash N_{q^{\prime} t}\right)=\left|\lambda_{\varphi\left(p^{\prime} t\right)}(U \backslash U)-\lambda_{\varphi\left(p^{\prime} t\right)}\left(N_{p^{\prime} t} \backslash N_{q^{\prime} t}\right)\right|<\varepsilon\right)$. Thus $\lambda_{\varphi(p)}\left(N_{p} \backslash N_{q}\right)<4 \varepsilon$ and since $\varepsilon$ was arbitrary, $\lambda_{\varphi(p)}\left(N_{p} \backslash N_{q}\right)=0$. So $(p, q) \in$ K.

To show that $K$ is closed in $X_{m} \times X_{m}$, let $\left(p_{n}, q_{n}\right)$ be a net in $K$ converging 
to a point $(p, q)$ in $X_{m} \times X_{m}$. Given any $\varepsilon>0$, take $U \in \mathscr{N}\left(N_{p}\right)$ and $V \in \mathcal{R}\left(N_{q}\right)$ such that $\lambda_{\varphi(p)}\left(U \backslash N_{p}\right)<\varepsilon$ and $\lambda_{\varphi(p)}\left(V \backslash N_{q}\right)<\varepsilon$, and so (i) $\left|\lambda_{\varphi(p)}(U \backslash V)-\lambda_{\varphi(p)}\left(N_{p} \backslash N_{q}\right)\right|<\varepsilon$. Now take $\left(p_{n}, q_{n}\right)$ in $K$ with $N_{p_{n}} \subseteq U$, $N_{q_{n}} \subseteq V, \lambda_{p\left(p_{n}\right)}\left(U \backslash N_{p_{n}}\right)<\varepsilon, \lambda_{\varphi\left(p_{n}\right)}\left(V \backslash N_{q_{n}}\right)<\varepsilon$, (and so (ii) $\mid \lambda_{\varphi\left(p_{n}\right)}(U \backslash V)-$ $\left.\lambda_{\varphi\left(p_{n}\right)}\left(N_{p_{n}} \backslash N_{q_{n}}\right) \mid<\varepsilon\right)$; (iii) $\left|\lambda_{\varphi(p)}(U \backslash V)-\lambda_{\varphi\left(p_{n}\right)}(U \backslash V)\right|<\varepsilon$. Since $\left(p_{n}, q_{n}\right)$ $\in K$, (iv) $\lambda_{\varphi\left(p_{n}\right)}\left(N_{p_{n}} \backslash N_{q_{n}}\right)=0$. Thus $\lambda_{\varphi(p)}\left(N_{p} \backslash N_{q}\right)<3 \varepsilon$, and since $\varepsilon$ was arbitrary, $\lambda_{(p)}\left(N_{p} \backslash N_{q}\right)=0$. So $(p, q) \in K$.

The proof of the additional remark is trivial.

(1.3) COROLLARY. If $X$ is metric, then

$$
\lambda_{\varphi\left(x_{0}\right)}\left(N_{x_{0}}\right)=\lambda_{\varphi\left(x_{0}\right)}\left(\cap\left\{N_{x}: x \in K\left(x_{0}\right)\right\}\right) \text {, }
$$

for $x_{0} \in X_{m}$.

Proof. Let $\left\{x_{i}\right\}_{1}^{\infty}$ be a countable dense subset of $K\left(x_{0}\right)$. Then since $N$ is closed $\cap\left\{N_{x}: x \in K\left(x_{0}\right)\right\}=\cap\left\{N_{x_{i}}: i=1,2, \ldots\right\}$.

(1.4) Corollary. Suppose $x_{0} \in X_{m}, A$ is a Borel set contained in $\theta^{-1}\left(\varphi\left(x_{0}\right)\right)$, and $N=\operatorname{cls}\left(\left(\left\{x_{0}\right\} \times A\right) T\right)$. Then for $x \in K\left(x_{0}\right), \lambda_{\varphi\left(x_{0}\right)}\left(A \cap N_{x}\right)$ $=\lambda_{\varphi\left(x_{0}\right)}(A)\left(\right.$ i.e. $\left.\lambda_{\varphi\left(x_{0}\right)}\left(A \backslash N_{x}\right)=0\right)$. If in addition $A=B \cap C$ where $B$ is a Borel set contained in the support of $\lambda_{\varphi\left(x_{0}\right)}$ with $\lambda_{\varphi\left(x_{0}\right)}(B)=1$ and $C$ is a nonempty open set, then $A \subseteq N_{x}$.

Proof. Note $N_{x_{0}} \supseteq A$, so $\lambda_{\varphi\left(x_{0}\right)}\left(A \backslash N_{x}\right) \leqslant \lambda_{\varphi\left(x_{0}\right)}\left(N_{x_{0}} \backslash N_{x}\right)=0$ and since $\left(A \cap N_{x}\right) \cup\left(A \backslash N_{x}\right)=A$, we see that $\lambda_{\varphi\left(x_{0}\right)}\left(A \cap N_{x}\right)=\lambda_{\varphi\left(x_{0}\right)}(A)$. If $A=B$ $\cap C$, then $0=\lambda_{\left(x_{0}\right)}\left(A \backslash N_{n}\right)=\lambda_{\varphi\left(x_{0}\right)}\left(B \cap\left(C \backslash N_{x}\right)\right)=\lambda_{\varphi\left(x_{0}\right)}\left(C \backslash N_{x}\right)$, but $C$ $\backslash N_{x}$ is open and so must be disjoint from the support of $\lambda_{\varphi\left(x_{0}\right)}$ and thus $(B \cap C) \backslash N_{x}=\varnothing$.

(1.5) Theorem. Suppose $X_{m}$ is a Borel subset of $X,(X=Y, \theta=\varphi)$, $(\varphi, \lambda) \in \mathcal{\delta}$, and for all $x$ in $X_{m} \cap B(x), \lambda_{\varphi(x)}\left(X_{m} \cap B(x)\right)=1$, where $B(x)$ is the support of $\lambda_{\varphi(x)}$. Then for $x \in X_{m} \cap B(x), Q_{m}(\varphi)(x)=S_{m}(\varphi)(x)$ and has the form $x^{\prime} \in Q_{m}(\varphi)(x)$ iff there exist nets $t_{n}$ in $T$ and $x_{n}$ in $X_{m} \cap B(x)$ such that $x_{n} \rightarrow x, x_{n} t_{n} \rightarrow x, x^{\prime} t_{n} \rightarrow x$. So for each $x^{\prime}$ in $X_{m}$,

$$
\begin{aligned}
B\left(x^{\prime}\right) & \cap S_{m}(\varphi)\left(x^{\prime}\right)=B\left(x^{\prime}\right) \cap Q_{m}(\varphi)\left(x^{\prime}\right) \\
& =X_{m} \cap\left(\cap \left\{\operatorname{cls}\left(\alpha T\left(x^{\prime}\right) \cap X_{m} \cap B\left(x^{\prime}\right)\right):\right.\right.
\end{aligned}
$$

$$
\left.\alpha=V \times V \text { where } V \text { is a neighborhood of } x_{0} \text { in } X\right\} \text { ) }
$$

$$
\text { for any fixed } x_{0} \text { in } X_{m} \text {. }
$$

Proof. Let $x \in X_{m} \cap B(x), x^{\prime} \in S_{m}(\varphi)(x)$, and $V$ an open neighborhood of $x$ in $X$. Let

$$
N=\operatorname{cls}\left(\left[\left\{x^{\prime}\right\} \times\left(V \cap X_{m} \cap B(x)\right)\right] T\right) .
$$


Then $N_{x} \supseteq V \cap X_{m} \cap B(x)$ by Corollary 1.4, since $x \in K\left(x^{\prime}\right)$ and $B(x)=$ $B\left(x^{\prime}\right)$. So $(x, x) \in N$ and there exist $t_{V}$ in $T$ and $x_{V}$ in $V \cap X_{m} \cap B(x)$ such that $x^{\prime} t_{V} \in V$ and $x_{V} t_{V} \in V$. This gives the desired net on the directed set consisting of open sets directed by inclusion. Clearly then $\left(x, x^{\prime}\right) \in Q_{m}(\varphi)$.

(1.6) Corollary. Suppose in addition that $x_{0} \in X_{m}, X$ has a countable neighborhood base at $x_{0}, X_{m}$ is a dense $G_{\delta}$ subset of $X$, and $S_{m}(\varphi)=R_{m}(\varphi)$. Then for each $x^{\prime}$ in $X_{m}$, the set $P_{m}(x) \cap X_{m} \cap B\left(x^{\prime}\right)$ is a dense $G_{8}$ subset of $X_{m} \cap B\left(x^{\prime}\right)$.

Proof. The corollary follows easily from the observation that $P_{m}\left(x^{\prime}\right) \cap X_{m}$ $\cap B\left(x^{\prime}\right)=\cap\left\{\alpha T\left(x^{\prime}\right) \cap X_{m} \cap B\left(x^{\prime}\right): \alpha=V \times V\right.$ where $V$ is a neighborhood of $x_{0}$ in $X$ \} for any fixed $x_{0}$ in $X_{m}$.

(1.7) Proposition. Suppose $(\varphi, \lambda) \in \delta, X$ is metric, $S_{m}(\varphi)=R_{m}(\varphi)$, and $\lambda_{\varphi(x)}\left(X_{m}\right)=1$ for $x \in X_{m}$. Then $\lambda_{\varphi(x)}\left(P_{m}(x)\right)=1$ and $\lambda_{\varphi(x)} \times \lambda_{\varphi(x)}\left(P_{m}\right)=1$ for $x$ in $X_{m}$.

Proof. Fix $x_{0}$ in $X_{m}$. Since $X$ is metric, $X$ has a countable neighborhood base, $\mathcal{T}$, at $x_{0}$, and $R(\varphi)(x) \cap P_{m}(x)=\bigcap\left\{\alpha T(x) \cap R_{m}(\varphi)(x): \alpha=V \times V\right.$ with $V \in \mathcal{V}\}$. Note $P_{m}(x)$ and $P_{m}$ are $G_{\delta}$ sets and so are measurable.

Now $\alpha T \cap R(\varphi)$ is an open invariant set in $R(\varphi)$, so $N=R(\varphi) \backslash \alpha T$ is a closed invariant set in $R(\varphi)$. By 1.2 and 1.3, since $X$ is metric

$$
\lambda_{\varphi(x)}\left(N_{x}\right)=\lambda_{\varphi(x)}\left(\cap\left\{N_{x^{\prime}}: x^{\prime} \in S_{m}(\varphi)(x)=R_{m}(\varphi)(x)\right\}\right)
$$

for $x$ in $X_{m}$. Note $\lambda_{\varphi(x)}(A)=\lambda_{\varphi(x)}\left(A \cap X_{m}\right)-\lambda_{\varphi(x)}\left(A \backslash X_{m}\right)=\lambda_{\varphi(x)}\left(A \cap X_{m}\right)$ for any Borel subset $A$ of $X$, since $\lambda_{\varphi(x)}\left(X_{m}\right)=1$. So if $\lambda_{\varphi(x)}\left(N_{x}\right) \neq 0$, there exists $x^{*}$ in $X_{m} \cap\left(\cap\left\{N_{x^{\prime}}: x^{\prime} \in R_{m}(\varphi)(x)\right\}\right)$. So $x^{*} \in N_{x^{*}}$, that is $\left(x^{*}, x^{*}\right) \in$ $N=R(\varphi) \backslash \alpha T$, which is a contradiction since $\alpha T$ contains the diagonal of $X_{m} \times X_{m}$. Thus

$$
\lambda_{\varphi(x)}\left(\alpha T(x) \cap R_{m}(\varphi)(x)\right)=\lambda_{\varphi(x)}\left(R_{m}(\varphi)(x) \backslash N_{x}\right)=1 .
$$

So $\lambda_{\varphi(x)}\left(R(\varphi)(x) \cap P_{m}(x)\right)=1$. Since the support of $\lambda_{\varphi(x)}$ is contained in $R(\varphi)(x)$, there is no loss in writing $\lambda_{\varphi(x)}\left(P_{m}(x)\right)=1$ for $x$ in $X_{m}$.

By Fubini's Theorem, $\lambda_{\varphi(x)} \times \lambda_{\varphi(x)}\left(P_{m}\right)=1$ for $x$ in $X_{m}$.

(1.8) Lemad. Suppose $(\theta, \lambda) \in \mathcal{S}, X$ is minimal (so $X=X_{m}, S(\varphi)=S_{m}(\varphi)$, $Z$ is minimal), $X$ and $Y$ are metric, and the natural projection $\pi: X \rightarrow X / S(\varphi)$ is open. Fix $x$ in $X$. Let $E(x)=\{y \in B(x):(x / S(\varphi), y)$ has dense orbit in $\left.(X / S(\varphi)){ }^{z} Y\right\}$ where $B(x)$ is the support of $\lambda_{\varphi(x)}$, and let $D(x)=\{y \in$ $B(x):(x, y)$ has dense orbit in $\left.X \circ^{Z} Y\right\}$. If $E(x)$ is a dense subset of $B(x)$, then $D(x)$ is a dense $G_{\delta}$ subset of $B(x)$. If $\lambda_{\varphi(x)}(E(x))=1$, then $\lambda_{\varphi(x)}(D(x))=$ 1. If $E(x)$ is a dense subset of $B(x)$ but $X$ and $Y$ are not necessarily metric, then we can conclude that $X \bullet^{Z} Y$ is topologically transitive. 
Proof. Fix $x$ in $X$. Let $\left\{U_{i}\right\},\left\{V_{i}\right\}$ be countable families of open sets in $X, Y$ respectively such that the set of $U_{i} \circ^{z} V_{i}=\left(U_{i} \times V_{i}\right) \cap\left(X \circ^{z} Y\right)$ is a countable base of nonempty open sets for the topology on $X \circ^{z} Y$. Fix $i$. Let $W$ be any open subset of $B(x)$ and $N=\operatorname{cls}((\{x\} \times x) T)$. Then $\left\{x^{\prime}\right\} \times W$ $\subseteq N$ for all $x^{\prime} \in S(\varphi)(x)$ by Corollary 1.4. So $S(\varphi)(W) \times W \subseteq N$. Now there exist $w$ in $W$ with $(x / S(\varphi), w)$ having dense orbit. Also $\left(U_{i} / S(\varphi)\right) \circ{ }^{z} V_{i}$ is open by assumption and nonempty since $U_{i} \circ^{z} V_{i} \neq \varnothing$. So there exists $t$ in $T$ with $(x / S(\varphi), w) t \in\left(U_{i} / S(\varphi)\right) \circ{ }^{z} V_{i}$. Then for some $x^{\prime} \in S(\varphi)(x), x^{\prime} t \in U_{i}$. Thus $N \cap U_{i} \times V_{i} \neq \varnothing$, so there exist $s$ in $T$ and $w^{\prime}$ in $W$ with $(x, w) s \in U_{i} \circ{ }^{z} V_{i}$. Now since $W$ was an arbitrary open set in $B(x)$, the set $A_{i}=\left\{a \in B(x):(x, a) t \in U_{i} \times V_{i}\right.$ for some $t$ in $\left.T\right\}$ is dense in $B(x)$, clearly it is open in $B(x)$. Let $y \in \cap_{1}^{\infty} A_{i}$, then $(x, y)$ has dense orbit in $X \circ^{z} Y$. So $D(x)=\cap_{1}^{\infty} A_{i}$ is a dense $G_{\delta}$ subset of $B(x)$.

Now suppose $\lambda_{\varphi(x)}(E(x))=1$ and $\lambda_{\varphi(x)}\left(A_{i}\right) \neq 1$ for some $i$. Then $\lambda_{\varphi(x)}(Y)$ $\left.A_{i}\right) \neq 0$. Let $N=\operatorname{cls}\left(\left[\{x\} \times\left(B(X) \backslash A_{i}\right)\right] T\right)$ and $C=\bigcap\left\{N_{x^{\prime}}: x^{\prime} \in S(\varphi)(x)\right\}$, then $\lambda_{\varphi(x)}(C) \neq 0$, by 1.3. Now $S(\varphi)(x) \times C \subseteq N$ and $(x / S(\varphi), c) t \in$ $\left(U_{i} / S(\varphi)\right) \times V_{i}$ for some $t$ in $T$ and $c$ in $C$ since $\lambda_{\varphi(x)}(C) \neq 0$ and $\lambda_{\varphi(x)}(E(x))$ $=1$. So there exists $x^{\prime}$ in $S(\varphi)(x)$ with $\left(x^{\prime}, c\right) t \in U_{i} \circ z V_{i}$. So $N \cap\left(U_{i} \times\right.$ $\left.V_{i}\right) \neq \varnothing$ and there exist $s$ in $T$ and $b$ in $B(x) \backslash A_{i}$ with $(x, b) s \in U_{i} \times V_{i}$. But then $b \in A_{i}$ by the definition of $A_{i}$, a contradiction. Thus $\lambda_{\varphi(x)}\left(A_{i}\right)=1$ and $\lambda_{\varphi(x)}(D(x))=1$.

To prove the last statement, let $U \circ z V$ be a nonempty open invariant set where $U$ and $V$ are open in $X$ and $Y$ respectively, we wish to show that $\left(U \circ{ }^{z} V\right) T$ is dense. Let $W$ be any relative open subset of $B(X)$, then $N=\operatorname{cls}(\{x\} \times W) T)$ has nonempty intersection with $U \bullet^{z} V$ and there exist $s$ in $T$ and $w^{\prime}$ in $W$ with $\left(x, w^{\prime}\right) s \in U \circ^{z} V$. So there exists an open neighborhood $W^{\prime}$ of $w^{\prime}$ relative to $B(x)$ with $\left(\{x\} \times W^{\prime}\right) s \subset U \circ^{z} V$. Then $X \circ^{z} Y=\operatorname{cls}\left(\left(\{x\} \times W^{\prime}\right) T\right) \subset \operatorname{cls}((U \circ z V) T)$.

(1.9) Corollary. Suppose $X$ and $Y$ are minimal and metric, the natural projection $X \rightarrow X / S(\varphi)$ is open, $(\theta, \lambda) \in \mathcal{S}$, and $X / S(\varphi) \perp^{z} Y / S(\theta)$. Fix $X$ in $X$ and let $D(x)=\left\{y \in B(x):(x, y)\right.$ has a dense orbit in $\left.X \circ{ }^{z} Y\right\}$ where $B(x)$ is the support of $\lambda_{\varphi(x)}$. Then $D(x)$ is a dense $G_{8}$ subset of $B(x)$ and $\lambda_{\varphi(x)}(D(x))=1$. In the nonmetric case we can conlcude that $X \circ^{z} Y$ is topologically transitive.

Proof. Note $X / S(\varphi){ }^{Z} Y$ is a disjoint union of minimal sets and, by 3.7 of [G], $X / S(\varphi) \rightarrow Z$ has a section; so by $1.8, X / S(\gamma) \circ Z Y$ has a point with dense orbit and thus is minimal (or see 3.8 of [W]). This implies $E(x)=$ $B(x)$. So the hypothesis of 1.8 is satisfied and the corollary follows.

Example 3.2.1 shows that the assumption that $X \rightarrow X / S(\varphi)$ is open cannot always be dropped. Example 3.2.2 shows one case in which 1.9 holds without 
$X \rightarrow X / S(\varphi)$ being open. Note this answers a conjecture of Glasner, [G],

(1.10) Lemma. Suppose $Z$ is a singleton, $Y$ supports an invariant Borel probability measure, $\lambda, X$ and $Y$ are metric, and $S_{m}(\varphi)=X_{m} \times X_{m}$. Then for $X$ in $X_{m}$, the set $D(x)=\{y \in Y:(x, y)$ has dense orbit in $X \times Y\}$ is a dense $G_{\delta}$ subset of $Y$. If also $\lambda\left(Y_{m}\right)=1$, then $\lambda(D(x))=1$.

Proof. (This proof is similar to that of Lemma 1.8.) Fix $x$ in $X_{m}$. Let $\left\{U_{i}\right\}$, $\left\{V_{i}\right\}$ be countable families of open sets in $X, Y$ respectively such that the set of $U_{i} \times V_{i}$ is a countable base of nonempty sets for the topology on $X \times Y$. Fix $i$. Let $W$ be any open subset of $Y$ and $N=\operatorname{cls}(\{x\} \times W) T)$. Then $\left\{x^{\prime}\right\} \times W \subseteq N$ for all $x^{\prime} \in S_{m}(\varphi)(x)$ by Corollary 1.4. So $X_{m} \times W \subseteq N$. So $X \times W \subseteq N$. Now there exists $w$ in $W$ with dense orbit, so for some $t$ in $T$ and $x^{\prime}$ in $X,\left(x^{\prime}, w\right) t \in U_{i} \times V_{i}$. So $N \cap\left(U_{i} \times V_{i}\right) \neq \varnothing$ and there exist $s$ in $T$ and $w^{\prime}$ in $W$ such that $\left(x, w^{\prime}\right) s \in U_{i} \times V_{i}$. Now since $W$ was an arbitrary open set in $Y$, the set $A_{i}=\left\{a \in Y:(x, a) t \in U_{i} \times V_{i}\right.$ for some $t$ in $\left.T\right\}$ is dense in $Y$, clearly it is open in $Y$. Then $D(x)=\cap_{1}^{\infty} A_{i}$ is a dense $G_{\delta}$ subset of $Y$.

Now suppose $\lambda\left(Y_{m}\right)=1$ and $\lambda\left(A_{i}\right) \neq 1$. Then $\lambda\left(Y \backslash A_{i}\right) \neq 0$. Let $N=$ $\operatorname{cls}\left(\left\{\{x\} \times\left(Y \backslash A_{i}\right)\right] T\right)$ and $C=\bigcap\left\{N_{x^{\prime}}: x^{\prime} \in S_{m}(\varphi)(x)\right\}=\bigcap\left\{N_{x^{\prime}}: x^{\prime} \in\right.$ $\left.X_{m}\right\}=\cap\left\{N_{x^{\prime}}: x^{\prime} \in X\right\}$ since $X_{m}$ is dense in $X$ and $N$ is closed. Then $\lambda(C) \neq 0$. Now $X \times C \subseteq N$, and $c t \in V_{i}$ for some $t$ and $c$ in $C$ since $\lambda(C) \neq 0$ and $\lambda\left(Y_{m}\right)=1$. So there exists $x^{\prime}$ in $X$ with $\left(x^{\prime}, c\right) t \in U_{i} \times V_{i}$ and thus $N \cap\left(U_{i} \times V_{i}\right) \neq \varnothing$. So there exist $s$ in $T$ and $y$ in $Y \backslash A_{i}$ with $(x, y) s \in$ $U_{i} \times V_{i}$. But then $y \in A_{i}$ by definition, a contradiction. Thus $\lambda\left(A_{i}\right)=1$ and $\lambda(D(x))=1$.

The following generalizes a result of Peleg $[P]$.

(1.11) Proposition. Suppose $Z$ is a singleton, $X$ and $Y$ are minimal and metric, $Y$ has an invariant Borel probability measure, $\lambda$, and $X / S(\varphi) \perp Y / S(\theta)$. Fix $x$ in $X$. Then the set $D(x)=\{y \in Y:(x, y)$ has dense orbit in $X \times Y\}$ is a dense $G_{8}$ subset of $Y$ and $\lambda(D(x))=1$.

Proof. Note $X / S \perp Y$, by 1.8 since $X / S$ has an invariant measure (or see 3.8 of [W] or 14 of [P]).

The remainder of the proof is similar to that of Lemma 1.8. Fix $x$ in $X$. Let $\left\{U_{i}\right\},\left\{V_{i}\right\}$ be countable families of open sets in $X, Y$ respectively such that the set of $U_{i} \times V_{i}$ is a countable base of nonempty open sets for the topology on $X \times Y$. Fix $i$. Let $W$ be any open set of $Y$ and $N=\operatorname{cls}((\{x\} \times W) T)$. Then $\left\{x^{\prime}\right\} \times W \subseteq N$ for all $x^{\prime} \in S(\varphi)(x)$ by Corollary 1.4. So $S(\varphi)(x) \times W$ $\subseteq N$. Now $U_{i} / S(\varphi)$ has nonempty interior since $X$ is minimal, and so $(x / S(\varphi), w) t \in\left(U_{i} / S(\varphi)\right) \times V_{i}$ for some $t$ in $T$ since $X / S(\varphi) \perp Y$. So $\left(x^{\prime}, w\right) t \in U_{i} \times V_{i}$ for some $x^{\prime}$ in $S(\varphi)(x)$. So $N \cap\left(U_{i} \times V_{i}\right) \neq \varnothing$ and 
therefore there exist $s$ in $T$ and $w^{\prime}$ in $W$ with $\left(x, w^{\prime}\right) s \in U_{i} \times V_{i}$. Now since $W$ was arbitrary, the set $A_{i}=\left\{a \in Y:(x, a) t \in U_{i} \times V_{i}\right.$ for some $t$ in $\left.T\right\}$ is an open dense subset of $B$. Thus $D(x)=\cap_{1}^{\infty} A_{i}$ is a dense $G_{\delta}$ subset of $Y$.

Now suppose $\lambda\left(A_{i}\right) \neq 1$. Then $\lambda\left(Y \backslash A_{i}\right) \neq 0$. Let $N=\operatorname{cls} \llbracket\{x\} \times(Y \backslash$ $\left.\left.A_{i}\right)\right] T$ ) and $C=\cap\left\{N_{x^{\prime}}: x^{\prime} \in S(\varphi)(x)\right\}$, then $\lambda(C) \neq 0$ and $C \neq \varnothing$. Now $S(\varphi)(x) \times C \subseteq N$ and for $c$ in $C,(x / S(\varphi), c) t \in U_{i} / S(\varphi) \times V_{i}$ for some $t$ in $T$, since $X / S(\varphi) \perp Y$ and $U_{i} / S(\varphi)$ has nonempty interior. So there exists $x^{\prime}$ in $S(\varphi)(x)$ with $\left(x^{\prime}, c\right) t \in U_{i} \times V_{i}$. So $N \cap\left(U_{i} \times V_{i}\right) \neq \varnothing$ and there exist $s$ in $T$ and $y$ in $Y \backslash A_{i}$ with $(x, y) s \in U_{i} \times V_{i}$. But then $y \in A_{i}$ by the definition of $A_{i}$, a contradiction. Thus $\lambda\left(A_{i}\right)=1$ and $\lambda(D(x))=1$.

(1.12) Remark. If $X$ is minimal and metric, $(\theta, \lambda) \in \mathcal{S}(X=Y, \varphi=\theta)$, $S(\varphi)=R(\varphi)$, and $\varphi$ is open, then for $x$ in $X, D(x)=\left\{x^{\prime} \in B(x):\left(x, x^{\prime}\right)\right.$ has dense orbit in $X \circ^{z} X$ \} is a dense $G_{\delta}$ subset of $B(x)$ and $\lambda_{\varphi(x)}(D(x))=1$. Moreover the transformation group $W_{n}=X \circ^{z} X \circ^{z} X \circ z \ldots \circ{ }^{z} X(n$ times) is point-transitive for each integer $n$, by 1.8. As a consequence $P_{m}\left(\varphi_{n}\right)$ is dense in $R\left(\varphi_{n}\right)$ where $\varphi_{n}: W_{n} \rightarrow Z$, since $R\left(\varphi_{n}\right)=W_{2 n}$ and $P_{m}\left(\varphi_{n}\right)=$ $\left\{\left(w, w^{\prime}\right) \in W_{n} \circ z W_{n}=W_{2 n}: w, w^{\prime}\right.$ are transitive points in $W_{n}$ and there exists a net $t_{i}$ in $T$ with $\left.\left(w, w^{\prime}\right) t_{i} \rightarrow(w, w)\right\}$ contains the transitive points in $W_{2 n}$.

(1.13) Proposmon. Suppose $Z$ is a singleton, $X$ is minimal and has an invariant Borel probability measure $\lambda, X$ is metric, $S_{X}=X \times X$, and $W_{n}=X$ $X \cdots \times X$ (n-times). Then for each $w$ in $\left(W_{n}\right)_{m}$ (the set of transitive points in $\left.W_{n}\right)$, the set $D(w)=\left\{x \in X:(w, x) \in\left(W_{n+1}\right)_{m}\right\}$ is a dense $G_{\delta}$ subset of $X$.

PRoof. $S_{m}\left(\varphi_{n}\right)=\left(W_{n}\right)_{m} \times\left(W_{n}\right)_{m}$ since $P_{m}\left(\varphi_{n}\right)$ is dense in $R\left(\varphi_{n}\right)=\left(W_{n}\right)$ $\times\left(W_{n}\right)$. The proposition follows from Lemma 1.10 upon simultaneously replacing $Y$ by $X$ and $X$ by $W_{n}$ and noting that then $Y_{m}=Y$.

2.

(2.1) Proposition. Suppose $\varphi: X \rightarrow Z$ has $D(\varphi)$ dense in $R(\varphi), X$ is minimal, $Z^{\prime}$ is minimal, and $\psi: Z^{\prime} \rightarrow Z$ is a proximal homomorphism. Let $X^{\prime}$ be the unique minimal set in $X \circ^{z} Z^{\prime}$ and let $\varphi^{\prime}$ and $\psi^{\prime}$ be the projections of $X^{\prime}$ onto $Z^{\prime}$ and $X$ respectively then

(1) If $\left(x, x^{*}\right) \in Q(\varphi)$, then there exists $z$ in $Z^{\prime}$ with $\left((x, z),\left(x^{*}, z\right)\right) \in$ $Q\left(\varphi^{\prime}\right)$.

(2) $S(\varphi)=\left\{\left(x, x^{*}\right) \in R(\varphi):\left(x u, x^{*} u\right) \in Q(\varphi)\right\}$ for some and thus every idempotent $u$ in $J$.

(3) $S\left(\varphi^{\prime}\right)=\left\{\left(x^{\prime}, x_{1}^{\prime}\right) \in R\left(\varphi^{\prime}\right):\left(x^{\prime} u, x_{1}^{\prime} u\right) \in Q\left(\varphi^{\prime}\right)\right\}$ for some and thus every idempotent $u$ in $J$.

(4) If $\left(x, x^{*}\right) \in S(\varphi)$, then $\left(\left(x, z^{\prime}\right),\left(x^{*}, z^{\prime}\right)\right) \in S\left(\varphi^{\prime}\right)$ for some $z^{\prime}$ in $Z^{\prime}$.

(5) Suppose $u \in J$ and $x_{0} \in X$, with $x_{0} u=x_{0}$. Then there exists a compact 
nonempty subset $B$ of $R(\varphi)\left(x_{0}\right)$ such that if $x \in R(\varphi)\left(x_{0}\right), x u=x, x^{\prime} \in B$, and $\left(x, x^{\prime}\right) \in S(\varphi)$, then $\left(x, x^{\prime}\right) \in Q(\varphi)$ and moreover there exist nets $t_{n}$ in $T$ and $x_{n}$ in $B$ with $x_{n} \rightarrow x, x_{n} t_{n} \rightarrow x, x^{\prime} t_{n} \rightarrow x$. So for $x^{\prime}$ in $X, B \cap S(\varphi) x^{\prime}=B \cap$ $Q(\varphi)\left(x^{\prime}\right)=\cap\left\{\operatorname{cls}\left(\alpha T\left(x^{\prime}\right) \cap B\right): \alpha=V \times V\right.$ where $V$ is a neighborhood of $x_{1}$ in $X$ \} for any fixed $x_{1}$ in $X$.

(6) If $S(\varphi)=R(\varphi)$ and $x_{i} u \in R(\varphi)\left(x_{0} u\right)$ for $i=1,2,3, \ldots, u \in J$, then there exist a nonempty compact subset $B$ of $R(\varphi)\left(x_{0} u\right)$ and a dense $G_{8}$ subset $F$ of $B$ such that $\left(x_{i}, f\right) \in P$ for $f$ in $F$ and $i=0,1,2, \ldots$

Proof of (1). Suppose $\left(x, x^{*}\right) \in Q(\varphi)$. Then there exist nets $t_{n}$ in $T$ and $\left(x_{n}, x_{n}^{*}\right) \in R(\varphi)$ such that $\left(x_{n}, x_{n}^{*}\right) \rightarrow\left(x, x^{*}\right)$ and $\left(x_{n}, x_{n}^{*}\right) t_{n} \rightarrow(x, x)$. Now since $D(\varphi)$ is dense in $R(\varphi)$, there exists a net $z_{n}^{\prime}$ in $Z^{\prime}$ with $\left(\left(x_{n}, z_{n}^{\prime}\right),\left(x_{n}^{*}, z_{n}^{\prime}\right)\right)$ $\in R\left(\varphi^{\prime}\right)$. We may assume $z_{n}^{\prime}$ and $z_{n}^{\prime} t_{n}$ converge. Thus (1) follows with $z^{\prime}=\lim z_{n}^{\prime}$.

Proof of (2). See 2.4 of [MW] or 2.6 .2 of $\left[\mathrm{V}_{2}\right]$.

Proof of (3). If $\left(\left(x, z^{\sim}\right),\left(x^{*}, z^{\sim}\right)\right) \in S\left(\varphi^{\prime}\right)$, then

$$
\left((x u, z \sim u),\left(x^{*} u, z \sim u\right)\right) \in S\left(\varphi^{\prime}\right) \subseteq S\left(\psi \circ \varphi^{\prime}\right)=S\left(\varphi \circ \psi^{\prime}\right) .
$$

So $\left(x u, x^{*} u\right) \in S(\varphi)$. Then $\left(x u, x^{*} u\right) \in Q(\varphi)$ by (2) and $\left(\left(x u, z^{*}\right)\right.$, $\left.\left(x^{*} u, z^{*}\right)\right) \in Q\left(\varphi^{\prime}\right)$ for some $z^{*} \in Z^{\prime}$. Then $\left(\left(x u, z^{*} u\right),\left(x^{*} u, z^{*} u\right)\right) \in Q\left(\varphi^{\prime}\right)$ and (3) follows since $z^{*} u=z_{\sim} u$.

Proof of (4). If $\left(x, x^{*}\right) \in S(\varphi)$ and $u \in J$, then $\left(x u, x^{*} u\right) \in Q(\varphi)$ and so there exists $z$ in $Z^{\prime}$ with $\left((x u, z),\left(x^{*} u, z\right)\right) \in Q\left(\varphi^{\prime}\right)$. So $\left((x u, z u),\left(x^{*} u, z u\right)\right) \in$ $Q\left(\varphi^{\prime}\right)$. Also there exists $z^{\prime}$ in $Z^{\prime}$ with $\left(\left(x, z^{\prime}\right),\left(x^{*}, z^{\prime}\right)\right) \in R\left(\varphi^{\prime}\right)$. Then $\left(\left(x u, z^{\prime} u\right),\left(x^{*} u, z^{\prime} u\right)\right) \in Q\left(\varphi^{\prime}\right)$ since $Z^{\prime}$ is a proximal extension of $Z$ implies $z^{\prime} u=z u$. Thus $\left(\left(x, z^{\prime}\right),\left(x^{*}, z^{\prime}\right)\right) \in S(\varphi)$ by $(3)$.

Proop of (5). (5) is a special case of 2.10 of [MW]. The proof here is completely different. Let $Z^{\prime}$ be $Z \sim$ as in 4.1 of [G]. So $\varphi^{\prime}$ has a section. Given $u \in J$ and $x_{0} \in X$ with $x_{0} u=x_{0}$, take $z \in Z^{\prime}$ with $\left(x_{0}, z\right) \in X^{\prime}$ and $z u=z$. Then take $B\left(x_{0}, z\right)$ as in 1.5. Note $B\left(x_{0}, z\right) \subseteq R(\varphi)\left(x_{0}\right) \times\{z\}$. Clearly (5) holds with $B=\left\{x \in X:(x, z) \in B\left(x_{0}, z\right)\right\}$.

Proof of (6). Follows easily from the proof of (5).

In 3.1 of [V], it is shown that for $\varphi: X \rightarrow Y$ with $X$ metric there exists a residual set $A$ of points $y$ in $Y$ such that $\varphi$ is open at each point of $\varphi^{-1}(y)$. In 3.3 of [G], it is shown that if, in addition to $X$ metric, $(\varphi, \lambda) \in \delta$, then there exists a residual set $B$ of points $y$ in $Y$ such that $\varphi^{-1}(y)$ equals the support $B_{y}$

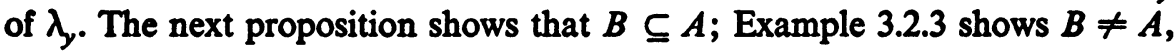
in general.

(2.2) Proposition. Suppose $\varphi: X \rightarrow Z,(\varphi, \lambda) \in \mathcal{S}$, and $x$ is in the support of $\lambda_{\varphi(x)}$. Then $\varphi$ is open at $x$.

Proof. Suppose $\varphi$ is not open at $x$ and $x \in B(x)=$ support of $\lambda_{\varphi(x)}$. Then 
there exists an open neighborhood $V$ of $x$ such that $\varphi(x) \notin \operatorname{int}(\varphi(V))$. Then there exists $t$ in $T$ with $\lambda_{y t}(V)>0$ and $\varphi^{-1}(y) t \cap V=\varnothing$. This is a contradiction.

3.

(3.1) Note. Example 2.2 of $[\mathrm{M}]$ is an example of an action on the torus $X$ that has the almost periodic points in $X \times X$ dense in $X \times X$ but does not have an invariant measure.

(3.2) EXAMPLES. The following examples are based on 2.1 of [M]. Note the examples will be nonmetric; however, metric examples could be obtained by letting $(Y, S)$ in 2.1 of $[M]$ be a countable dense subgroup $S$ of the Cantor group provided with the discrete topology acting on the Cantor set by right multiplication. The verifications of the properties of the following examples are straightforward.

(1) Define $g^{\prime}$ by

$$
g^{\prime}(y, w)= \begin{cases}0 & \text { if } h(y)>h(w), \\ 1 & \text { if } h(y)<h(w) \text { and } h(y) \text { is even, } \\ 2 & \text { if } h(y)<h(w) \text { and } h(y) \text { is odd. }\end{cases}
$$

Then $\varphi_{g^{\prime}}: X_{g^{\prime}} \rightarrow Y$ has a RIM, $S\left(\varphi_{g^{\prime}}\right)=R\left(\varphi_{g^{\prime}}\right), \varphi_{g^{\prime}}$ is not open, and $R\left(\varphi_{g^{\prime}}\right)$ is not point-transitive.

(2) Define $g^{*}$ by

$$
g^{*}(y, w)= \begin{cases}0 & \text { if } h(y)>h(w) \text { or } h(y) \text { is odd, } \\ 1 & \text { if } h(y)<h(w) \text { and } h(y) \text { is even. }\end{cases}
$$

Then $R\left(\varphi_{g^{*}}\right)$ is point-transitive and $\varphi_{g^{*}}$ is not open.

(3) Define $f$ by

$$
f(y, w)= \begin{cases}0 & \text { if } h(y)>h(w), \\ 1 & \text { if } h(y)<h(w) .\end{cases}
$$

Then $\varphi_{f}$ has a section $\lambda, \varphi_{f}$ is open, and the support of $\lambda_{y_{0}}$ does not equal $\varphi^{-1}\left(y_{0}\right)$, where $\left(y_{0}, w_{0}\right)$ is the point of discontinuity of $f$.

4. For this section we refer the reader to III.3 of $\left[\mathbf{G}_{b}\right]$ for a sketch of a construction of a universal point-transitive transformation group $|L|$ with transitive point $x_{e}$ for a topological group $T$ with identity $e$. Note in $\left[G_{b}\right]$ the group acts on the left, whereas it acts on the right in this paper. Because of this the definition of left uniformly continuous functions should be changed by defining $f^{t}$ to be $f^{t}(s)=f(s t)$. Such a function is sometimes called right uniformly continuous, [K]; such functions are exactly the functions that are uniformly continuous with respect to the left uniform structure as defined in 
[HR]. Using 8.2 of [HR], we note that the mapping $t \rightarrow x_{e} t$ is a homeomorphism of $T$ onto $x_{e} T$; identifying $t$ with $x_{e} t$, we note that for any subset $S$ of $T$ and any neighborhood $W$ of the identity, int(cls $(S W))$ is an open set in $|L|$ containing cls $S$.

Let $M$ be a minimal right ideal in $|L|$. As sketched in 3.1 of $\left[\mathrm{G}_{\mathrm{b}}\right], T$ is amenable iff $M$ has an invariant measure.

(4.1) Proposinon. Let $T$ be an amenable group, $M$ be the universal minimal set for $T$, and $u$ be an idempotent in $M$. Suppose $B$ is a syndetic subset of $T$ and $W$ is an open neighborhood of $e$ in $T$. Then there exists a neighborhood $V$ of $u / Q$ in the compact Hausdorff group $M / Q$ such that $t \in W^{-1} B^{-1} B U B^{-1} B W$ for all neighborhoods $U$ of $e$ in $T$ and for all $t$ in $T$ with ut $/ Q$ in $V$. If $B$ is discretely syndetic, then there exists a neighborhood $V$ of $u / Q$ in $M / Q$ such that $t \in B^{-1} B U B^{-1} B$ for all neighborhoods $U$ of $e$ in $T$ and all $t$ in $T$ with $u t / Q$ in $V$.

Proof. By definition $T=B K$ for some compact subset $K$ of $T$ and so $|L|=\operatorname{cls}(B K)$. Then note that $M \cap \operatorname{cls}(B) \neq \varnothing$. So the set $A=M \cap$ int(cls $(B W)$ ) is a nonempty open (in $M$ ) subset of $M$. (When $B$ is discretely syndetic, $B F=T$, where $F$ is finite, let $C=M \cap$ cls $B$ and note $C F=M$, so $A=\operatorname{int}_{M}(C) \subseteq$ cls $B$ is a nonempty open subset of $M$.) Now $A / Q$ is a subset of $M / Q$ with nonempty interior, int $(A / Q)$, since $M$ is minimal. Let $m \in A$ with $M / Q$ in $\operatorname{int}(A / Q)$ and let $u$ be an idempotent in $M$ with $m u=m$. Then $m^{-1} A$ is a neighborhood of $u$ and $V=\operatorname{int}\left(m^{-1} A / Q\right)$ is a neighborhood of $u / Q$ (since $M / Q$ is a regular minimal set). Now suppose $t \in T$ with $u t / Q \in V$. Then $(u t, p) \in Q$ for some $p$ in $m^{-1} A$. So $(m t, m p) \in$ $Q$ and by 1.5 there exist $q$ in $M$ and $s$ in $T$ with $q$ in $A$ and $q s, m t s$ in $\operatorname{cls}(B U)$ for any neighborhood $U$ of $e$ in $T$. Also $m \in A$. We note that $q$ and $m$ can be approximated by $b_{1} w_{1}$ and $b_{2} w_{2}$ so that $b_{1} w_{1} s$ and $b_{2} w_{2} t s$ are in $\operatorname{cls}(B U)$. So $b_{1} w_{1} s U \cap B U \neq \varnothing$ and $b_{2} w_{2} t s U \cap B U \neq \varnothing$. So $t \in$ $W^{-1} B^{-1} B U U^{-1} U U^{-1} B^{-1} B W$. To get the stated result simply note that we could have used a $U_{0}$ with $U_{0} U_{0}^{-1} U_{0} U_{0}^{-1} \subseteq U$ in the proof. When $B$ is discretely syndetic, $A \subseteq$ cls $B$ and so for any neighborhood $U$ of $e$ in $T$, $\operatorname{cls}(B U)$ is a neighborhood of $A$ in $M$ and $q$ and $m$ may be approximated by $b_{1}$ and $b_{2}$. Then $t \in B^{-1} B U U^{-1} U U^{-1} B^{-1} B$.

ReMark. Motivated by 3.12 of [EK], we prove the following.

(4.3) Corollary. Let $T$ be abelian, $B$ syndetic, and $W$ a neighborhood of $E$ in $T$. Then there exist $b_{0}$ in $B$ and a neighborhood $V$ of $u / Q$ in $M / Q$ such that $t \in W B^{-1} B B b_{0}^{-1}$ for all $t$ with $u t / Q$ in $V$.

Proof. We will merely sketch the portion of the proof that parallels that of 4.1. When $A=M \cap \operatorname{int}(\operatorname{cls}(B W))$ there is $a t_{0}=b_{0} w_{0}$ with $(u / Q) b_{0} w_{0} \in$ $\operatorname{int}(A / Q)$. Take $V=\operatorname{int}(A / Q)\left(b_{0} w_{0}\right)^{-1}$. If $(u t, p) \in Q$, then $\left(u t t_{0}, p t_{0}\right) \in Q$ 
and $\left(v t t_{0}, p t_{0}\right) \in Q$ where $v$ is an idempotent in $M$ with $p v=p$. Take nets $q_{n}$ in $M$ and $s_{n}$ in $T$ with $q_{n} \rightarrow p t_{0}, q_{n} s_{n} \rightarrow p t_{0}, v t t_{0} s_{n} \rightarrow p t_{0}$. Note $p t_{0}$ is in $A$. We may assume $q_{n} v=q_{n}$ since $M v$ is dense in $M$, and we may assume $r=\lim s_{n}$ $\in M$ since $v s_{n}$ has the same effect as $s_{n}$ on $q_{n}$ and $v t t_{0}$ (let $a_{\lambda} \rightarrow v$ and use the iterated limit theorem). Now $v t t_{0} s_{n} \rightarrow r t t_{0}$, so $r t t_{0}=p t_{0}$ and $s_{n} t t_{0} \rightarrow r t t_{0}=p t_{0}$. So we may fix an $n$ such that $s_{n} t t_{0} \in A$ and also $q_{n} \in A$ and $q_{n} s_{n} \in A$. Approximate $q_{n}$ by $b_{1} w_{1}$ so that $b_{1} w_{1} s_{\mathrm{n}} \in \operatorname{cls}(B W)$. Then $s_{n} t b_{0} w_{0}=s_{n} t t_{0} \in$ $B W W^{-1}$ and $b_{1} w_{1} s_{n} \in B W W^{-1}$. So $t \in W W^{-1} B^{-1} B W W^{-1} w_{0}^{-1} b_{0}^{-1}$. The corollary clearly follows.

(4.4) EXAMPLE. The $b_{0}^{-1}$ in the above cannot be dropped as the following example shows. Let $T$ be the discrete group of integers and let $T$ act on the unit circle $K$ in the complex plane by irrational rotation, so $(K, T)$ is minimal. Let $V$ be a neighborhood of 1 in $K$ and $k \in K$ such that $V \cap V^{3} k=\varnothing$. Let $B=\left\{t \in T: 1 t \in V_{k}\right\}$. Then $V \cap B^{-1} B B \subseteq V \cap V^{3} k=\varnothing$.

\section{REFERENCES}

[E] R. Ellis, Lectures on topological dynamics, Benjamin, New York, 1969. MR 42 \#2463.

[EK] R. Ellis and H. Keynes, Bohr compactifications and a result of Folner, Israel J. Math. 12 (1972), 314-330.

[F] E. Følner, Generalization of a theorem of Bogoliouboff to topological abelian groups, Math. Scand. 2 (1954), 5-18.

, Note on a generalization of a theorem of Bogoliouboff, Math. Scand. 2 (1954), 224-226.

[FG] H. Furstenberg and S. Glasner, On the existence of isometric extensions (preprint).

[G] S. Glasner, Relatively invariant measures, Pacific J. Math. 58 (1975), 393-410.

$\left[G_{\mathbf{b}}\right]$ , Proximal flows, Lecture Notes in Math. Vol. 517, Springer-Verlag, New York, 1976.

[HR] E. Hewitt and K. Ross, Abstract harmonic analysis. I, Springer-Verlag, New York, 1963.

[E] A. W. Knapp, Decompasition theorem for bounded uniformly continuous functions on a group, Amer. J. Math. 88 (1966), $902-914$.

[M] D. McMahon, Weak mixing and a note on a structure theorem for minimal transformation groups, Illinois J. Math. 20 (1976), 186-197.

[MW] D. McMahon and T. S. Wu, On proximal and distal extensions of minimal sets, Bull. Inst. Math. Acad. Sinica 2 (1974), 93-107.

[P] R. Peleg, Weak disjointness of transformation groups, Proc. Amer. Math. Soc. 33 (1972), $165-170$.

[W] T. S. Wu, Notes on topological dynamics. I, Relative disjointness, relative regularity, and homomorphisms, Bull. Inst. Math. Acad. Sinica 2 (1974), 343-356.

[V] W. Veech, Point-distal flows, Amer. J. Math. 92 (1970), 205-242.

$\left[V_{2}\right]$ _ Topological dynamics, Bull. Amer. Math. Soc. 83 (1977), 775-830.

Department of Mathematical Sciences, OakLand University, Rochester, Michion 48063

Current address: Department of Mathematics, Arizona State University, Tempe, Arizona 85281 